\title{
Stability of Talagrand's Gaussian transport-entropy inequality via the Föllmer process
}

\author{
Dan Mikulincer*
}

April 27, 2021

\begin{abstract}
We establish a dimension-free improvement of Talagrand's Gaussian transport-entropy inequality, under the assumption that the measures satisfy a Poincaré inequality. We also study stability of the inequality, in terms of relative entropy, when restricted to measures whose covariance matrix trace is smaller than the ambient dimension. In case the covariance matrix is strictly smaller than the identity, we give dimension-free estimates which depend on its eigenvalues. To complement our results, we show that our conditions cannot be relaxed, and that there exist measures with covariance larger than the identity, for which the inequality is not stable, in relative entropy. To deal with these examples, we show that, without any assumptions, one can always get quantitative stability estimates in terms of relative entropy to Gaussian mixtures. Our approach gives rise to a new point of view which sheds light on the hierarchy between Fisher information, entropy and transportation distance, and may be of independent interest. In particular, it implies that the described results apply verbatim to the log-Sobolev inequality and improve upon some known estimates in the literature.
\end{abstract}

\section{Introduction}

Talagrand's Gaussian transport-entropy inequality, first proved in [30], states that for any measure $\mu$ in $\mathbb{R}^{d}$, with a finite second moment matrix,

$$
\mathcal{W}_{2}^{2}(\mu, \gamma) \leq 2 \mathrm{D}(\mu \| \gamma)
$$

Here, $\gamma$ denotes the standard Gaussian measure on $\mathbb{R}^{d}$, with density

$$
\varphi(x)=\frac{1}{(\sqrt{2 \pi})^{d}} e^{\frac{-\|x\|_{2}^{2}}{2}} .
$$

The distances involved in the inequality are, $\mathrm{D}(\mu \| \gamma)$, the relative entropy, defined by

$$
\mathrm{D}(\mu \| \gamma)=\int_{\mathbb{R}^{d}} \ln \left(\frac{d \mu}{d \gamma}\right) d \mu,
$$

*Weizmann Institute of Science. Supported by an Azrieli foundation fellowship. 
and $\mathcal{W}_{p}(\mu, \gamma)$ is the $L_{p}$-Wasserstein distance (with $L_{2}$ cost function),

$$
\mathcal{W}_{p}(\mu, \gamma)=\sqrt[p]{\inf _{\pi} \int_{\mathbb{R}^{2 d}}\|x-y\|_{2}^{p} d \pi(x, y)},
$$

where the infimum runs over all measures on $\mathbb{R}^{2 d}$ whose marginal laws onto the first and last $d$ coordinates are $\mu$ and $\gamma$. Since this fundamental inequality tensorizes, it holds in any dimension. Using this quality, the inequality was shown to imply a sharp form of the dimension-free concentration of measure phenomenon in Gaussian space. The reader is referred to [20,23,31] for further information on the topic. By setting the measure $\mu$ to be a translation of $\gamma$, we can see that the inequality is tight and that, in particular, the constant 2 in (1) cannot be improved. One, in fact, may show that these examples account for the only equality cases of (1). We are thus led to consider the question of stability of the inequality. Consider the deficit

$$
\delta_{\text {Tal }}(\mu):=2 \mathrm{D}(\mu \| \gamma)-\mathcal{W}_{2}^{2}(\mu, \gamma) .
$$

Suppose that $\delta_{\text {Tal }}(\mu)$ is small. In this case, must $\mu$ be necessarily close to a translate of $\gamma$ ?

A first step towards answering this question, which serves as a starting point for the current work, was given in [15] (see also [22]), where it was shown that there exists a numerical constant $c>0$, such that if $\mu$ is centered,

$$
\delta_{\text {Tal }}(\mu) \geq c \min \left(\frac{\mathcal{W}_{1,1}^{2}(\mu, \gamma)}{d}, \frac{\mathcal{W}_{1,1}(\mu, \gamma)}{\sqrt{d}}\right)
$$

Here, $\mathcal{W}_{1,1}$ stands for the $L_{1}$-Wasserstein distance with $L_{1}$-cost function. The inequality was later improved in [8], and $\frac{\mathcal{W}_{1,1}(\mu, \gamma)}{\sqrt{d}}$ was replaced by the larger quantity $\mathcal{W}_{1}(\mu, \gamma)$. One could hope to improve this result in several ways; First, one may consider stronger notions of distance than $\mathcal{W}_{1,1}$, like relative entropy. Indeed by Jensen's inequality and (1),

$$
\frac{\mathcal{W}_{1,1}^{2}(\mu, \gamma)}{d} \leq \mathcal{W}_{2}^{2}(\mu, \gamma) \leq 2 \mathrm{D}(\mu \| \gamma)
$$

Second, note that for product measures, $\delta_{\mathrm{Tal}}(\mu)$ grows linearly in $d$, while the RHS of (2) may grow like $\sqrt{d}$ (this remains true for the improved result, found in [8]). The dimensionfree nature of (1) suggests that the dependence on the dimension in (2) should, hopefully, be removed. The goal of the present work is to identify cases in which (2) may be improved. Specifically, we will be interested in giving dimension-free stability bounds with respect to the relative entropy distance. We will also show that, without further assumptions on the measure $\mu$, (2) cannot be significantly improved.

This work adds to a recent line of works which explored dimension-free stability estimates for functional inequalities in the Gaussian space, such as the log-Sobolev inequality $[2,12,15,16$, $24]$, the Shannon-Stam inequality $[9,13]$ and the Gaussian isoperimetric inequality $[1,7,26]$.

\section{Results}

In our first main result, we restrict our attention to the subclass of probability measures which satisfy a Poincaré inequality. A measure $\mu$ is said to satisfy a Poincaré inequality with constant $\mathrm{C}_{\mathrm{p}}(\mu)$, if for every smooth function $g: \mathbb{R}^{d} \rightarrow \mathbb{R}$,

$$
\int_{\mathbb{R}^{d}} g^{2} d \mu-\left(\int_{\mathbb{R}^{d}} g d \mu\right)^{2} \leq \mathrm{C}_{\mathrm{p}}(\mu) \int_{\mathbb{R}^{d}}\|\nabla g\|_{2}^{2} d \mu,
$$


where we implicitly assume that $\mathrm{C}_{\mathrm{p}}(\mu)$ is the smallest constant for which this inequality holds. If $\mu$ satisfies such an inequality, then, in some sense, $\mu$ must be regular. Indeed, $\mu$ must have finite moments of all orders. For such measures we prove:

Theorem 1. Let $\mu$ be a centered measure on $\mathbb{R}^{d}$ with finite Poincaré constant $\mathrm{C}_{\mathrm{p}}(\mu)<\infty$. Then

$$
\delta_{\text {Tal }}(\mu) \geq \min \left(\frac{1}{4}, \frac{\left(\mathrm{C}_{\mathrm{p}}(\mu)+1\right)\left(2-2 \mathrm{C}_{\mathrm{p}}(\mu)+\left(\mathrm{C}_{\mathrm{p}}(\mu)+1\right) \ln \left(\mathrm{C}_{\mathrm{p}}(\mu)\right)\right)}{\left(\mathrm{C}_{\mathrm{p}}(\mu)-1\right)^{3}}\right) \mathrm{D}(\mu \| \gamma) .
$$

Note that as the deficit is invariant to translations, there is no loss in generality in assuming that $\mu$ is centered. Furthermore, the Poincaré constant tensorizes, in the sense that for any two measures $\nu$ and $\mu, \mathrm{C}_{\mathrm{p}}(\nu \otimes \mu)=\max \left(\mathrm{C}_{\mathrm{p}}(\nu), \mathrm{C}_{\mathrm{p}}(\mu)\right)$. So, if $\mu$ is a product measure $\mathrm{C}_{\mathrm{p}}(\mu)$ does not depend on the dimension and we regard it as a dimensionless quantity. For a more applicable form of the result we may use the inequality

$$
\min \left(\frac{1}{4}, \frac{(x+1)(2-2 x+(x+1) \ln (x))}{(x-1)^{3}}\right) \geq \frac{\ln (x+1)}{4 x},
$$

valid for $x>0$, to get

$$
\delta_{\text {Tal }}(\mu) \geq \frac{\ln \left(\mathrm{C}_{\mathrm{p}}(\mu)+1\right)}{4 \mathrm{C}_{\mathrm{p}}(\mu)} \mathrm{D}(\mu \| \gamma) .
$$

Theorem 1 should be compared with Theorem 1 in [15] and Theorem 7 in [13] which give similar stability estimates, involving the Poincaré constant, for the log-Sobolev and Shannon-Stam inequalities.

Regarding the conditions of the theorem; as will be shown in Section 2 below, there exists a measure $\mu$ for which $\delta_{\text {Tal }}(\mu)$ may be arbitrarily close to 0 , while $\mathcal{W}_{2}(\mu, \gamma)$ remains bounded away from 0 . Thus, in order to establish meaningful stability results, in relative entropy, it is necessary to make some assumptions on the measure $\mu$.

In case the measure $\mu$ does not satisfy a Poincaré inequality, we provide estimates in terms of its covariance matrix. It turns out, that if $\operatorname{Cov}(\mu)$ is strictly smaller than the identity, at least in some directions, we may still produce a dimension-free bound for $\delta_{\text {Tal }}(\mu)$.

Theorem 2. Let $\mu$ be a centered measure on $\mathbb{R}^{d}$ and let $\left\{\lambda_{i}\right\}_{i=1}^{d}$ be the eigenvalues of $\operatorname{Cov}(\mu)$, counted with multiplicity. Then

$$
\delta_{\text {Tal }}(\mu) \geq \sum_{i=1}^{d} \frac{2\left(1-\lambda_{i}\right)+\left(\lambda_{i}+1\right) \log \left(\lambda_{i}\right)}{\lambda_{i}-1} \mathbb{1}_{\left\{\lambda_{i}<1\right\}} .
$$

Remark that for $0<x<1$, the function $g(x):=\frac{2(1-x)+(x+1) \log (x)}{x-1}$ is positive and that it is a decreasing function of $x$. Also, it can be verified that $g^{\prime}$ is actually concave on this domain, from which we may see $g(x) \geq \frac{1}{6}(x-1)^{2}$. Thus, if $\operatorname{Cov}(\mu) \preceq \mathrm{I}_{d}$, then the Theorem implies the weaker result

$$
\delta_{\text {Tal }}(\mu) \geq \frac{1}{6}\left\|\mathrm{I}_{d}-\operatorname{Cov}(\mu)\right\|_{H S}^{2},
$$

where $\|\cdot\|_{H S}$, stands for the Hilbert-Schmidt norm. In line with the above discussion, we may regard $\left\|\operatorname{Cov}(\mu)-\mathrm{I}_{d}\right\|_{H S}^{2}$ as a certain distance between $\mu$ and the standard Gaussian. Theorem 3 in [12] gives a similar estimate for the log-Sobolev inequality. Indeed, our methods are based 
on related ideas.

If $\operatorname{Cov}(\mu)=I_{d}$, Theorem 2 does not give any new insight beyond (1). The next result applies, among others, to this case.

Theorem 3. Let $\mu$ be a centered measure on $\mathbb{R}^{d}$, such that $\operatorname{Tr}(\operatorname{Cov}(\mu)) \leq d$. Then

$$
\delta_{\text {Tal }}(\mu) \geq \min \left(\frac{\mathrm{D}(\mu \| \gamma)^{2}}{6 d}, \frac{\mathrm{D}(\mu \| \gamma)}{4}\right) .
$$

As opposed to the previous two results, Theorem 3 is not dimension-free and is directly comparable to (2). Under the assumption $\operatorname{Tr}(\operatorname{Cov}(\mu)) \leq d$, by using (3) we may view the theorem as a strengthening of (2). We should also comment that by Pinsker's inequality ( [10]), relative entropy induces a stronger topology than the $\mathcal{W}_{1}$ metric. On the other hand, (2) holds in greater generality than Theorem 3 as it makes no assumptions on the measure $\mu$. It is then natural to ask whether one can relax the conditions of the theorem. We give a negative answer to this question.

Theorem 4. Fix $d \in \mathbb{N}$ and let $\xi>d$. There exist a sequence of centered measures $\mu_{k}$ on $\mathbb{R}^{d}$ such that:

- $\lim _{k \rightarrow \infty} \operatorname{Tr}\left(\operatorname{Cov}\left(\mu_{k}\right)\right)=\xi$.

- $\lim _{k \rightarrow \infty} \delta_{\text {Tal }}\left(\mu_{k}\right)=0$.

- $\liminf _{k \rightarrow \infty} \mathcal{W}_{2}^{2}\left(\mu_{k}, \gamma\right) \geq \xi-d>0$.

Thus, even for one dimensional measures, in order to obtain general stability estimates in relative entropy or even in the quadratic Wasserstein distance, the assumption $\operatorname{Tr}(\operatorname{Cov}(\mu)) \leq d$ is necessary.

The counterexample to stability, guaranteed by Theorem 4, may be realized as a Gaussian mixture. In fact, as demonstrated by recent works ( $[6,9,12])$, Gaussian mixtures may serve as counterexamples to stability of several other Gaussian functional inequalities. This led the authors of [12] to note that if a measure $\mu$ saturates the log-Sobolev inequality, then it must be close, in $L_{2}$-Wasserstein distance, to some Gaussian mixture. We show that this is also true, in relative entropy, for Talagrand's inequality.

Theorem 5. Let $\mu$ be a centered measure on $\mathbb{R}^{d}$. Then there exists another measure $\nu$ with $\operatorname{Cov}(\nu) \preceq \operatorname{Cov}(\mu)$, such that if $\delta_{\text {Tal }}(\mu) \geq d$,

$$
\delta_{\text {Tal }}(\mu) \geq \frac{\mathrm{D}(\mu \| \nu * \gamma)}{6},
$$

and if $\delta_{\mathrm{Tal}}(\mu)<d$,

$$
\delta_{\mathrm{Tal}}(\mu) \geq \frac{1}{3 \sqrt{3}} \frac{\mathrm{D}(\mu \| \nu * \gamma)^{\frac{3}{2}}}{\sqrt{d}} .
$$

Note that, in light of Theorem 4, the above theorem is not true without the convolution, and we cannot, in general, replace $\nu * \gamma$ by $\gamma$.

For our last result, define the Fisher information of $\mu$, relative to $\gamma$, as

$$
\mathrm{I}(\mu \| \gamma):=\int_{\mathbb{R}^{d}}\left\|\nabla \ln \left(\frac{d \mu}{d \gamma}\right)\right\|_{2}^{2} d \mu .
$$


Gross' log-Sobolev inequality ( [21]) states that

$$
\mathrm{I}(\mu \| \gamma) \geq 2 \mathrm{D}(\mu \| \gamma)
$$

For this we define the deficit as

$$
\delta_{\mathrm{LS}}(\mu)=\mathrm{I}(\mu \| \gamma)-2 \mathrm{D}(\mu \| \gamma) .
$$

As will be described in Section 3 below, our approach draws a new connection between Talagrand's and the log-Sobolev inequalities. One benefit of this approach is that all of our results apply verbatim to the log-Sobolev inequality. Some of the results improve upon existing estimates in the literature. We summarize those in the following corollary.

Corollary 6. Let $\mu$ be a centered measure on $\mathbb{R}^{d}$. Then there exists a measure $\nu$ such that $\operatorname{Cov}(\nu) \preceq \operatorname{Cov}(\mu)$ and

$$
\delta_{\mathrm{LS}}(\mu) \geq \min \left(\frac{1}{3 \sqrt{3}} \frac{\mathrm{D}(\mu \| \nu * \gamma)^{\frac{3}{2}}}{\sqrt{d}}, \frac{\mathrm{D}(\mu \| \nu * \gamma)}{6}\right) .
$$

Moreover, if $\operatorname{Tr}(\operatorname{Cov}(\mu)) \leq d$ then

$$
\delta_{\mathrm{LS}}(\mu) \geq \min \left(\frac{\mathrm{D}(\mu \| \gamma)^{2}}{6 d}, \frac{\mathrm{D}(\mu \| \gamma)}{4}\right),
$$

The second point of the corollary is an improvement of Corollary 1.2 in [2] which shows, under the same hypothesis,

$$
\delta_{\mathrm{LS}}(\mu) \geq c \frac{\mathcal{W}_{2}^{4}(\mu, \gamma)}{d}
$$

for some universal constant $c>0$. The improved bound can actually be deduced from Theorem 1.1 in the same paper, but it does not seem to appear in the literature explicitly

The first point of Corollary 6 strengthens Theorem 7 in [12] which states, that for some measure $\nu$ :

$$
\delta_{\mathrm{LS}}(\mu) \geq \frac{1}{15} \frac{\mathcal{W}_{2}^{3}(\mu, \nu * \gamma)}{\sqrt{d}}
$$

Our proof closely resembles theirs, but our analysis yields bounds in the stronger relative entropy distance. The authors of [12] raise the natural question, whether the dependence on the dimension in (4) can be completely discarded. The same question is also relevant to $\delta_{\text {Tal }}(\mu)$. We do not know the answer to either of the questions, which seem related.

\section{Organization}

The remainder of the paper is organized as follows: In Section 2 we give a counter-example to stability of Talagrand's inequality, proving Theorem 4. Section 3 is devoted to explaining our method and proving some of its basic properties which will then be used in Section 4 to prove the stability estimates. Finally, in Section 5 we give an application of our results to Gaussian concentration inequalities.

\section{Acknowledgments}

We wish to thank Ronen Eldan, Max Fathi, Renan Gross, Emanuel Indrei and Yair Shenfeld for useful discussions and for their comments concerning a preliminary draft of this work. We are also grateful to the anonymous referee for carefully reading this paper and providing thoughtful comments. 


\section{A counterexample to stability}

In this section we show that one cannot expect any general stability result to hold if $\operatorname{Tr}(\operatorname{Cov}(\mu))>d$. We present a one-dimensional example, which may be easily generalized to higher dimensions. The following notations will be used in this section:

- For $\sigma^{2}>0, \gamma_{\sigma^{2}}$ denotes the law of the centered 1-dimensional Gaussian with variance $\sigma^{2}$.

- Fix $\xi>1$ and $k \in \mathbb{N}$, we set

$$
\mu_{k}:=\left(1-\frac{1}{k}\right) \gamma_{1}+\frac{1}{k} \gamma_{k(\xi-1)}
$$

Recall now the Kantorovich dual formulation (see [19,31], for example) of the $L_{2}$-Wasserstien distance. For $\nu$ and $\mu$ measures on $\mathbb{R}$, we have

$$
\mathcal{W}_{2}^{2}(\mu, \nu)=\sup _{g}\left\{\int_{\mathbb{R}} g(x) d \mu(x)-\int_{\mathbb{R}}(Q g)(x) d \nu(x)\right\}
$$

where the supremum runs over all measurable functions, and $Q g$ denotes the sup-convolution of $g$, namely

$$
Q g(x)=\sup _{y \in \mathbb{R}}\left\{g(y)-(x-y)^{2}\right\}
$$

Proof of Theorem 4. We first note that $\operatorname{Var}\left(\mu_{k}\right) \stackrel{k \rightarrow \infty}{\longrightarrow} \xi>1$. Towards understanding $\delta_{\text {Tal }}\left(\mu_{k}\right)$ we use the fact that relative entropy is convex with respect to mixtures of measures ( [10]), so

$$
\mathrm{D}\left(\mu_{k} \| \gamma\right) \leq \frac{1}{k} \mathrm{D}\left(\gamma_{k(\xi-1)} \| \gamma\right)=\frac{1}{2 k}(k(\xi-1)-1-\ln (k(\xi-1))) \leq \frac{\xi-1}{2} .
$$

To control the Wasserstein distance, define the functions

$$
g_{k}(x)= \begin{cases}0 & \text { if }|x|<\frac{\sqrt{k}}{\ln (k)} \\ \left(1-\frac{1}{\ln (k)}\right) x^{2} & \text { otherwise }\end{cases}
$$

The main idea is that as $k$ increases, $Q g_{k}$ vanishes in an ever expanding region, while growing slowly outside of the region. Formally, for $0 \leq x \leq \frac{\sqrt{k}}{\ln (k)}-\frac{\sqrt{k(\ln (k)-1)}}{\ln (k)^{\frac{3}{2}}}$, it holds that

$$
g_{k}\left(\frac{\sqrt{k}}{\ln (k)}\right)-\left(x-\frac{\sqrt{k}}{\ln (k)}\right)^{2}=\left(1-\frac{1}{\ln (k)}\right)\left(\frac{\sqrt{k}}{\ln (k)}\right)^{2}-\left(x-\frac{\sqrt{k}}{\ln (k)}\right)^{2} \leq 0 .
$$

and in particular, if $\frac{\sqrt{k}}{\ln (k)}<y$,

$$
g_{k}(y)-(x-y)^{2}<0
$$

which shows $Q g_{k}(x)=0$. There exists a constant $c>0$ such that

$$
\frac{\sqrt{k}}{\ln (k)}-\frac{\sqrt{k(\ln (k)-1)}}{\ln (k)^{\frac{3}{2}}} \geq c k^{\frac{1}{4}}
$$


which, combined with the previous observation shows that for $|x| \leq c k^{\frac{1}{4}}, Q g_{k}(x)=0$. If $|x|>c k^{\frac{1}{4}}$ it is standard to show $Q g_{k}(x) \leq \ln (k) x^{2}$. So,

$$
\int_{\mathbb{R}} Q g_{k}(x) d \gamma_{1}(x) \leq \ln (k) \int_{|x| \geq c k^{\frac{1}{4}}} x^{2} d \gamma_{1}(x)=\ln (k)\left(\frac{c \sqrt{2}}{\sqrt{\pi}} k^{\frac{1}{4}} e^{-\frac{c^{2} \sqrt{k}}{2}}+\int_{|x| \geq c k^{\frac{1}{4}}} d \gamma_{1}\right) \stackrel{k \rightarrow \infty}{\longrightarrow} 0,
$$

where the equality is integration by parts. Also, it is clear that

$$
\int_{\mathbb{R}} g_{k}(x) d \gamma_{1}(x) \stackrel{k \rightarrow \infty}{\longrightarrow} 0 .
$$

Now, if $\varphi$ denotes the density of the standard Gaussian, then by a change of variables we have

$$
\begin{aligned}
\frac{1}{k} \int_{\mathbb{R}} g_{k}(x) d \gamma_{k(\xi-1)}(x) & =\left(1-\frac{1}{\ln (k)}\right) \frac{1}{k} \int_{|x| \geq \frac{\sqrt{k}}{\ln (k)}} \frac{x^{2}}{\sqrt{k(\xi-1)}} \varphi\left(\frac{x}{\sqrt{k(\xi-1)}}\right) d x \\
& =\left(1-\frac{1}{\ln (k)}\right)(\xi-1) \int_{|y| \geq \frac{1}{\ln (k) \sqrt{\xi-1}}} y^{2} \varphi(y) d y \stackrel{k \rightarrow \infty}{\longrightarrow} \xi-1 .
\end{aligned}
$$

Combining the above displays with (5) we get,

$$
\begin{aligned}
\mathcal{W}_{2}^{2}\left(\mu_{k}, \gamma_{1}\right) & \geq \int_{\mathbb{R}} g_{k}(x) d \mu_{k}(x)-\int_{\mathbb{R}} Q g_{k}(x) d \gamma_{1}(x) \\
& =\left(1-\frac{1}{k}\right) \int_{\mathbb{R}} g_{k}(x) d \gamma_{1}(x)+\frac{1}{k} \int_{\mathbb{R}} g_{k}(x) d \gamma_{k(\xi-1)}(x)-\int_{\mathbb{R}} Q g_{k}(x) d \gamma_{1}(x) \stackrel{k \rightarrow \infty}{\longrightarrow} \xi-1 .
\end{aligned}
$$

Finally, from (6) we obtain

$$
\delta_{\mathrm{Tal}}\left(\mu_{k}\right)=2 \mathrm{D}\left(\mu_{k}|| \gamma_{1}\right)-\mathcal{W}_{2}^{2}\left(\mu_{k}, \gamma_{1}\right) \stackrel{k \rightarrow \infty}{\longrightarrow} 0
$$

We remark that, in light of Theorem 5, it would seem more natural to have as a counterexample a mixture of Gaussians with unit variance, as was done in [12] for the log-Sobolev inequality. However, (2) tells us that the situation in Talagrand's inequality is a bit more delicate, since the inequality is stable with respect to the $\mathcal{W}_{1}$ metric. Thus, as in the given example, a counterexample to stability (in the $\mathcal{W}_{2}$ metric or relative entropy) should satisfy $\lim _{k \rightarrow \infty} \mathcal{W}_{1}\left(\mu_{k}, \gamma\right)=0$, while $\liminf _{k \rightarrow \infty} \mathcal{W}_{2}\left(\mu_{k}, \gamma\right)>0$. keeping this in mind, it seems more straightforward to allow the second moments of the summands in the mixture to vary while keeping their means fixed at the origin. This is also very similar to the counterexample, obtained in [9], for the entropy power inequality. 


\section{The Föllmer process}

Our method is based on an entropy minimizing process, known in the literature as the Föllmer process. The high-level idea underlying this work is to use the process in order to embed a given measure as the terminal point of some martingale, in the Wiener space. This will induce a coupling between the measure and $\gamma$. As will be shown, the process also solves a variational problem, which turns out to yield a representation formula for the relative entropy. Combining these two properties will allow us to bound $\delta_{\text {Tal }}(\mu)$ from below.

The process appears in the works of Föllmer ( $[17,18])$. It was later used by Borell in [3] and Lehec in [25] to give simple proofs of various functional inequalities, including Talagrand's Gaussian transport-entropy inequality. Recently, the process was used in order to prove stability estimates for the Shannon-Stam ( [13]) and log-Sobolev ( [12]) inequalities. In this section, we present the relevant details concerning the process. The reader is referred to $[11,14,25]$ for further details and a more rigorous treatment. We will sketch the main ideas here for completeness.

Throughout this section we fix a measure $\mu$ on $\mathbb{R}^{d}$ with expectation 0 , a finite second moment matrix and a density $f$, relative to $\gamma$. Consider the Wiener space $C\left([0,1], \mathbb{R}^{d}\right)$ of continuous paths with the Borel sigma-algebra generated by the supremum norm $\|\cdot\|_{\infty}$. We endow $C\left([0,1], \mathbb{R}^{d}\right)$ with a probability measure $P$ and a process $B_{t}$ which is a Brownian motion under $P$. We will denote by $\omega$ elements of $C\left([0,1], \mathbb{R}^{d}\right)$ and by $\mathcal{F}_{t}$ the natural filtration of $B_{t}$. Define the measure $Q$ by

$$
\frac{d Q}{d P}(\omega)=f\left(\omega_{1}\right)
$$

$Q$ is absolutely continuous with respect to $P$, in which case, a converse to Girsanov's theorem implies that there exists a drift, $v_{t}$, adapted to $\mathcal{F}_{t}$, in the Wiener space, such that the process

$$
X_{t}:=B_{t}+\int_{0}^{t} v_{s}\left(X_{s}\right) d s
$$

has the same law as $Q$, and that, under $Q, X_{t}$ is a Brownian motion. In particular, by construction, $X_{1} \sim \mu$ and conditioned on $X_{1}, X_{t}$ serves a Gaussian bridge between 0 and $X_{1}$. Thus, by the representation formula for Brownian bridges

$$
X_{t} \stackrel{\text { law }}{=} t X_{1}+\sqrt{t(1-t)} G
$$

where $G$ is a standard Gaussian, independent from $X_{1}$. We call $v_{t}\left(X_{t}\right)$ the Föllmer drift and $X_{t}$ the Föllmer process. As $\mu$ and $\gamma$ are the laws of $X_{1}$ and $B_{1}$, it is now immediate that

$$
\mathrm{D}(Q \| P) \geq \mathrm{D}(\mu \| \gamma)
$$

A remarkable feature is that, since $\frac{d Q}{d P}$ depends only on the terminal points, the above is actually an equality and $\mathrm{D}(Q \| P)=\mathrm{D}(\mu \| \gamma)$. This implies that the drift, $v_{t}$, is a martingale (see Lemmas 10 and 11 in [25]).

We now use Girsanov's theorem ( [27, Theorem 8.6.3]) to rewrite $\frac{d Q}{d P}$ as an exponential martingale,

$$
\frac{d Q}{d P}(\omega)=\exp \left(-\int_{0}^{1} v_{t}(\omega) d X_{t}(\omega)+\frac{1}{2} \int_{0}^{1}\left\|v_{t}(\omega)\right\|_{2}^{2} d t\right) .
$$


Under $Q, X_{t}$ is a Brownian motion, so

$$
\mathrm{D}(Q \| P)=\int_{C\left([0,1], \mathbb{R}^{d}\right)} \ln \left(\frac{d Q}{d P}\right) d Q=\frac{1}{2} \int_{0}^{1} \mathbb{E}\left[\left\|v_{t}\left(X_{t}\right)\right\|_{2}^{2}\right] d t,
$$

which gives the formula

$$
\mathrm{D}(\mu \| \gamma)=\frac{1}{2} \int_{0}^{1} \mathbb{E}\left[\left\|v_{t}\left(X_{t}\right)\right\|_{2}^{2}\right] d t
$$

For simplicity, from now on, we suppress the dependence of $v_{t}$ on $X_{t}$. Combining the above with (9) shows that among all adapted drifts $u_{t}$ such that $\mu \sim B_{1}+\int_{0}^{1} u_{t} d t, v_{t}$ minimizes the energy in the following sense

$$
v_{t}=\arg \min _{u_{t}} \frac{1}{2} \int_{0}^{1} \mathbb{E}\left[\left\|u_{t}\right\|_{2}^{2}\right] d t
$$

Theorem 12 in [25] capitalizes on the structure of $\frac{d P}{d Q}$ to give an explicit representation of $v_{t}$ as

$$
v_{t}=\nabla \ln \left(P_{1-t} f\left(X_{t}\right)\right) \text {. }
$$

where $P_{1-t}$ denotes the heat semi-group. Since $v_{t}$ is a martingale, Itô's formula shows

$$
d v_{t}=\nabla v_{t} d B_{t}=\nabla^{2} \ln \left(P_{1-t} f\left(X_{t}\right)\right) d B_{t} .
$$

Lehec's proof of Talagrand's transport-entropy inequality relied on the fact that (7) induces a natural coupling between $\mu$ and $\gamma$ so that, by Jensen's inequality

$$
\mathcal{W}_{2}^{2}(\mu, \gamma) \leq \mathbb{E}\left[\left\|X_{1}-B_{1}\right\|_{2}^{2}\right] \leq \int_{0}^{1} \mathbb{E}\left[\left\|v_{t}\right\|_{2}^{2}\right] d t=2 \mathrm{D}(\mu \| \gamma)
$$

Our goal is to make this quantitative.

\subsection{The martingale approach}

As was demonstrated in [13] and [14] it is often easier to work with an equivalent martingale formulation of the Föllmer drift. Consider the Doob martingale $\mathbb{E}\left[X_{1} \mid \mathcal{F}_{t}\right]$. By the martingale representation theorem ( [27, Theorem 4.33]) there exists a uniquely-defined, adapted, matrix valued process $\Gamma_{t}$ which satisfies

$$
\mathbb{E}\left[X_{1} \mid \mathcal{F}_{t}\right]=\int_{0}^{t} \Gamma_{s} d B_{s}
$$

We claim that

$$
v_{t}=\int_{0}^{t} \frac{\Gamma_{s}-\mathrm{I}_{d}}{1-s} d B_{s}
$$


Indeed, by Fubini's theorem

$$
\begin{aligned}
\int_{0}^{1} \Gamma_{s} d B_{s}=\int_{0}^{1} \mathrm{I}_{d} d B_{s}+\int_{0}^{1}\left(\Gamma_{s}-\mathrm{I}_{d}\right) d B_{s} & =B_{1}+\int_{0}^{1} \int_{s}^{1} \frac{\Gamma_{s}-\mathrm{I}_{d}}{1-s} d t d B_{s} \\
& =B_{1}+\int_{0}^{1} \int_{0}^{t} \frac{\Gamma_{s}-\mathrm{I}_{d}}{1-s} d B_{s} d t .
\end{aligned}
$$

For the moment denote $\tilde{v}_{t}:=\int_{0}^{t} \frac{\Gamma_{s}-\mathrm{I}_{d}}{1-s} d B_{s}$. Since $v_{t}$ is a martingale $v_{t}-\tilde{v}_{t}$ is a martingale as well and the above shows that for every $t \in[0,1]$, almost surely,

$$
\int_{t}^{1}\left(v_{s}-\tilde{v}_{s}\right) d s \mid \mathcal{F}_{t}=0
$$

This implies the identity (14). In particular, from (12), $\Gamma_{t}$ turns out to be symmetric, which shows, using Itô's formula,

$$
2 \mathrm{D}(\mu \| \gamma)=\int_{0}^{1} \mathbb{E}\left[\left\|v_{t}\right\|_{2}^{2}\right] d t=\operatorname{Tr} \int_{0}^{1} \int_{0}^{t} \frac{\mathbb{E}\left(\Gamma_{s}-\mathrm{I}_{d}\right)^{2}}{(1-s)^{2}} d s d t=\operatorname{Tr} \int_{0}^{1} \frac{\mathbb{E}\left(\Gamma_{t}-\mathrm{I}_{d}\right)^{2}}{1-t} d t .
$$

Also, note that

$$
B_{1}+\int_{0}^{1}\left(\Gamma_{t}-\mathrm{I}_{d}\right) d B_{t}=\int_{0}^{1} \Gamma_{t} d B_{t} \sim \mu
$$

which implies

$$
\mathcal{W}_{2}^{2}(\mu, \gamma) \leq \operatorname{Tr} \int_{0}^{1} \mathbb{E}\left[\left(\Gamma_{t}-\mathrm{I}_{d}\right)^{2}\right] d t
$$

As $X_{1} \sim \mu$, from (12) we get

$$
\operatorname{Tr} \int_{0}^{1} \frac{\mathbb{E}\left[\left(\Gamma_{t}-\mathrm{I}_{d}\right)^{2}\right]}{(1-t)^{2}} d t=\mathbb{E}\left[\left\|v_{1}\right\|_{2}^{2}\right]=\int_{\mathbb{R}^{d}}\|\nabla \ln (f(x))\|_{2}^{2} d \mu(x)=\mathrm{I}(\mu \| \gamma) .
$$

Combining (15),(16),(17), we see a very satisfying connection between the log-Sobolev and Talagrand's transport-entropy inequalities, as

$$
\operatorname{Tr} \int_{0}^{1} \frac{\mathbb{E}\left[\left(\Gamma_{t}-\mathrm{I}_{d}\right)^{2}\right]}{(1-t)^{2}} d t \geq \operatorname{Tr} \int_{0}^{1} \frac{\mathbb{E}\left[\left(\Gamma_{t}-\mathrm{I}_{d}\right)^{2}\right]}{1-t} d t \geq \operatorname{Tr} \int_{0}^{1} \mathbb{E}\left[\left(\Gamma_{t}-\mathrm{I}_{d}\right)^{2}\right] d t,
$$

implies

$$
\mathrm{I}(\mu \| \gamma) \geq 2 \mathrm{D}(\mu \| \gamma) \geq \mathcal{W}_{2}^{2}(\mu, \gamma)
$$

In addition to its elegance, this representation can prove useful in the study of stability properties for those functional inequalities. We have the following representation for the deficits,

$$
\delta_{\mathrm{LS}}(\mu)=\operatorname{Tr} \int_{0}^{1} t \cdot \frac{\mathbb{E}\left[\left(\Gamma_{t}-\mathrm{I}_{d}\right)^{2}\right]}{(1-t)^{2}} d t,
$$




$$
\delta_{\text {Tal }}(\mu) \geq \operatorname{Tr} \int_{0}^{1} t \cdot \frac{\mathbb{E}\left[\left(\Gamma_{t}-\mathrm{I}_{d}\right)^{2}\right]}{1-t} d t .
$$

The above formulas are the key to Corollary 6.

Proof of Corollary 6. Note that by (18) and (19), any estimate on $\delta_{\text {Tal }}(\mu)$ which is achieved by bounding

$$
\operatorname{Tr} \int_{0}^{1} t \cdot \frac{\mathbb{E}\left[\left(\Gamma_{t}-\mathrm{I}_{d}\right)^{2}\right]}{1-t} d t
$$

from below will also imply a bound for $\delta_{\mathrm{LS}}(\mu)$. Since Theorem 3 and Theorem 5 are proved using this method, the corollary follows.

One should remark that, by using (12) and (14), we have the identification

$$
\nabla^{2} \ln \left(P_{1-t} f\left(X_{t}\right)\right)=\frac{\Gamma_{t}-\mathrm{I}_{d}}{1-t}
$$

Thus, all of the calculations above could have been done with respect to $v_{t}$, without appealing to the Doob martingale in (13).

However, we hope that by introducing the matrix $\Gamma_{t}$, we may shed some new light on the subject, which may be of independent use elsewhere. As an example, consider the bounds proved in [14, Theorem 6], for the rate of convergence in the entropic central limit theorem and in [12, Lemma 2] for stability of the Shannon-Stam inequality. In all cases, the proof of the quantitative estimates boils down to showing that the matrix $\Gamma_{t}$ is different than the identity, far from 0 .

We also feel that some calculations become more natural when dealing with the matrix $\Gamma_{t}$. As an easy example for the use of the above formulation, consider the case in which $\delta_{\text {Tal }}(\mu)=0$. By (19), it follows that, for every $t \in[0,1], \Gamma_{t}=\mathrm{I}_{d}$ almost surely. Thus, since $\mu \sim \int_{0}^{1} \Gamma_{t} d B_{t}, \mu$ must be the standard Gaussian, which is known to be the only centered equality case (Of-course one could also conclude that $f \equiv 1$, which yields the same result).

\subsection{Properties of the Föllmer process}

Our objective is now clear: In order to produce any stability estimates it will be enough to show, roughly speaking, that the process $\Gamma_{t}$ is far from $\mathrm{I}_{d}$, not too close to time 0 . In order to establish such claims we will use several other properties of the processes $\Gamma_{t}, v_{t}$, which we now state and prove. First, as in [14, Lemma 11] it is possible use (14) along with integration by parts to obtain the identity:

$$
\mathbb{E}\left[v_{t} \otimes v_{t}\right]=\frac{\mathbb{E}\left[\mathrm{I}_{d}-\Gamma_{t}\right]}{1-t}+\left(\operatorname{Cov}(\mu)-\mathrm{I}_{d}\right)
$$


Combining the fact that $v_{t}$ is a martingale with (14) we also see

$$
\begin{aligned}
\frac{d}{d t} \mathbb{E}\left[\left\|v_{t}\right\|_{2}^{2}\right] & =\operatorname{Tr} \frac{\mathbb{E}\left[\left(\mathrm{I}_{d}-\Gamma_{t}\right)^{2}\right]}{(1-t)^{2}} \\
& \geq \frac{1}{d}\left(\operatorname{Tr}\left(\frac{\mathbb{E}\left[\mathrm{I}_{d}-\Gamma_{t}\right]}{1-t}\right)\right)^{2}=\frac{\left(\mathbb{E}\left[\left\|v_{t}\right\|_{2}^{2}\right]-\operatorname{Tr}\left(\operatorname{Cov}(\mu)-\mathrm{I}_{d}\right)\right)^{2}}{d},
\end{aligned}
$$

where we have used Cauchy-Schwartz for the inequality. Using this we prove the following two lemmas:

\section{Lemma 1. It holds that}

$$
\frac{d}{d t} \mathbb{E}\left[\Gamma_{t}\right]=\frac{\mathbb{E}\left[\Gamma_{t}\right]-\mathbb{E}\left[\Gamma_{t}^{2}\right]}{1-t}
$$

Proof. Since $\Gamma_{t}$ is a symmetric matrix equation (14) implies

$$
\frac{d}{d t} \mathbb{E}\left[v_{t} \otimes v_{t}\right]=\frac{\mathbb{E}\left[\left(\mathrm{I}_{d}-\Gamma_{t}\right)^{2}\right]}{(1-t)^{2}}
$$

Combined with (20), this gives

$$
\frac{\mathbb{E}\left[\left(\mathrm{I}_{d}-\Gamma_{t}\right)^{2}\right]}{(1-t)^{2}}=\frac{d}{d t} \frac{\mathbb{E}\left[\mathrm{I}_{d}-\Gamma_{t}\right]}{1-t}=\frac{\mathbb{E}\left[\mathrm{I}_{d}-\Gamma_{t}\right]-(1-t) \frac{d}{d t} \mathbb{E}\left[\Gamma_{t}\right]}{(1-t)^{2}}
$$

Rearranging the terms yields the result.

Lemma 2. Suppose that $\operatorname{Tr}(\operatorname{Cov}(\mu)) \leq d$ and let $v_{t}$ be as defined above. Then:

- For $0 \leq t \leq \frac{1}{2}, \mathbb{E}\left[\left\|v_{t}\right\|_{2}^{2}\right] \leq \mathbb{E}\left[\left\|v_{1 / 2}\right\|_{2}^{2}\right] \frac{2 d}{\mathbb{E}\left[\left\|v_{1 / 2}\right\|_{2}^{2}\right](1-2 t)+2 d}$

- For $\frac{1}{2} \leq t \leq 1, \mathbb{E}\left[\left\|v_{t}\right\|_{2}^{2}\right] \geq \mathbb{E}\left[\left\|v_{1 / 2}\right\|_{2}^{2}\right] \frac{2 d}{\mathbb{E}\left[\left\|v_{1 / 2}\right\|_{2}^{2}\right](1-2 t)+2 d}$

Proof. Since $\operatorname{Tr}(\operatorname{Cov}(\mu)) \leq d,(21)$ gives

$$
\frac{d}{d t} \mathbb{E}\left[\left\|v_{t}\right\|_{2}^{2}\right] \geq \frac{\left(\mathbb{E}\left[\left\|v_{t}\right\|_{2}^{2}\right]\right)^{2}}{d}
$$

The unique solution to the differential equation

$$
g^{\prime}(t)=\frac{g(t)^{2}}{d}, \text { with inital condition } g\left(\frac{1}{2}\right)=\mathbb{E}\left[\left\|v_{1 / 2}\right\|_{2}^{2}\right],
$$

is given by

$$
g(t)=\mathbb{E}\left[\left\|v_{1 / 2}\right\|_{2}^{2}\right] \frac{2 d}{\mathbb{E}\left[\left\|v_{1 / 2}\right\|_{2}^{2}\right](1-2 t)+2 d} .
$$

The result follows by Gronwall's inequality 
To get a different type of inequality, but of similar flavor, recall (8),

$$
X_{t} \stackrel{\text { law }}{=} t X_{1}+\sqrt{t(1-t)} G
$$

where $G$ is a standard Gaussian, independent from $X_{1}$. Now, suppose that $\mu$ satisfies a Poincaré inequality with optimal constant $\mathrm{C}_{\mathrm{p}}(\mu)$. In this case $X_{t}$ satisfies a Poincaré inequality with a constant, smaller than, $t^{2} \mathrm{C}_{\mathrm{p}}(\mu)+t(1-t)$. This follows from the fact that the Poincare constant is sub-additive with respect to convolutions ( [4]) and that if $X \sim \nu$ and $a X \sim \nu_{a}$ for some $a \in \mathbb{R}$, then $\mathrm{C}_{\mathrm{p}}\left(\nu_{a}\right)=a^{2} \mathrm{C}_{\mathrm{p}}(\nu)$. Applying the Poincaré inequality to $v_{t}\left(X_{t}\right)$, we get

$$
\mathbb{E}\left[\left\|v_{t}\right\|_{2}^{2}\right] \leq\left(t^{2} \mathrm{C}_{\mathrm{p}}(\mu)+t(1-t)\right)\left[\left\|\nabla v_{t}\right\|_{2}^{2}\right]=\left(t^{2} \mathrm{C}_{\mathrm{p}}(\mu)+t(1-t)\right) \frac{d}{d t}\left[\left\|v_{t}\right\|_{2}^{2}\right],
$$

where the equality is due to the fact that $v_{t}$ is a martingale. Repeating the proof of Lemma 2 for the differential equation

$$
g(t)=\left(t^{2} \mathrm{C}_{\mathrm{p}}(\mu)+t(1-t)\right) g^{\prime}(t), \text { with inital condition } g\left(\frac{1}{2}\right)=\mathbb{E}\left[\left\|v_{1 / 2}\right\|_{2}^{2}\right],
$$

proves:

Lemma 3. Assume that $\mu$ has a finite Poincaré constant $\mathrm{C}_{\mathrm{p}}(\mu)<\infty$. Then, for $v_{t}$ defined as above:

- $\operatorname{For} 0 \leq t \leq \frac{1}{2}$,

$$
\mathbb{E}\left[\left\|v_{t}\right\|_{2}^{2}\right] \leq \mathbb{E}\left[\left\|v_{1 / 2}\right\|_{2}^{2}\right] \frac{\left(\mathrm{C}_{\mathrm{p}}(\mu)+1\right) t}{\left(\mathrm{C}_{\mathrm{p}}(\mu)-1\right) t+1}
$$

- For $\frac{1}{2} \leq t \leq 1$,

$$
\mathbb{E}\left[\left\|v_{t}\right\|_{2}^{2}\right] \geq \mathbb{E}\left[\left\|v_{1 / 2}\right\|_{2}^{2}\right] \frac{\left(\mathrm{C}_{\mathrm{p}}(\mu)+1\right) t}{\left(\mathrm{C}_{\mathrm{p}}(\mu)-1\right) t+1}
$$

\section{Stability for Talagrand's transportation-entropy inequal- ity}

We begin this section by showing two ways the Föllmer process may be used to establish quantitative stability estimates. As before, $\mu$ is a fixed measure on $\mathbb{R}^{d}$ with finite second moment matrix. $\Gamma_{t}$ and $v_{t}$ are defined as in the previous section. Fix $t_{0} \in[0,1]$, by (19), we see

$$
\delta_{\text {Tal }}(\mu) \geq t_{0} \operatorname{Tr} \int_{t_{0}}^{1} \frac{\mathbb{E}\left[\left(\mathrm{I}_{d}-\Gamma_{t}\right)^{2}\right]}{1-t} d t .
$$

Now, using (14), we obtain, by Fubini's theorem,

$$
\int_{t_{0}}^{1}\left(\mathbb{E}\left[\left\|v_{s}\right\|_{2}^{2}\right]-\mathbb{E}\left[\left\|v_{t_{0}}\right\|_{2}^{2}\right]\right) d s=\operatorname{Tr} \int_{t_{0}}^{1} \int_{t_{0}}^{s} \frac{\mathbb{E}\left[\left(\mathrm{I}_{d}-\Gamma_{t}\right)^{2}\right]}{(1-t)^{2}} d t d s=\operatorname{Tr} \int_{t_{0}}^{1} \frac{\mathbb{E}\left[\left(\mathrm{I}_{d}-\Gamma_{t}\right)^{2}\right]}{1-t} d t
$$

and

$$
\delta_{\text {Tal }}(\mu) \geq t_{0}\left(\int_{t_{0}}^{1} \mathbb{E}\left[\left\|v_{t}\right\|_{2}^{2}\right] d t-\left(1-t_{0}\right) \mathbb{E}\left[\left\|v_{t_{0}}\right\|_{2}^{2}\right]\right) \geq t_{0}\left(1-t_{0}\right)\left(2 \mathrm{D}(\mu \| \gamma)-\mathbb{E}\left[\left\|v_{t_{0}}\right\|_{2}^{2}\right]\right),
$$


where we have used (15) and the fact that $v_{t}$ is a martingale. Another useful bound will follow by applying (14) to rewrite (19) as

$$
\delta_{\text {Tal }}(\mu) \geq \operatorname{Tr} \int_{0}^{1} t(1-t) \cdot \frac{\mathbb{E}\left[\left(\Gamma_{t}-\mathrm{I}_{d}\right)^{2}\right]}{(1-t)^{2}} d t=\int_{0}^{1} t(1-t) \frac{d}{d t} \mathbb{E}\left[\left\|v_{t}\right\|_{2}^{2}\right] d t .
$$

Integration by parts then gives

$$
\delta_{\text {Tal }}(\mu) \geq \int_{0}^{1}(2 t-1) \mathbb{E}\left[\left\|v_{t}\right\|_{2}^{2}\right] d t
$$

At an informal level, the above formula becomes useful if one is able to show that $\mathbb{E}\left[\left\|v_{t}\right\|_{2}^{2}\right]$ is large for $t \geq \frac{1}{2}$ and small otherwise.

\subsection{Measures with a finite Poincaré constant}

We now assume that the measure $\mu$ has a finite Poincaré constant $\mathrm{C}_{\mathrm{p}}(\mu)<\infty$.

Proof of Theorem 1. First, suppose that $\mathbb{E}\left[\left\|v_{1 / 2}\right\|_{2}^{2}\right] \leq \mathrm{D}(\mu \| \gamma)$. In this case (23) shows

$$
\delta_{\text {Tal }} \geq \frac{1}{4} \mathrm{D}(\mu \| \gamma)
$$

Otherwise, $\mathbb{E}\left[\left\|v_{1 / 2}\right\|_{2}^{2}\right]>\mathrm{D}(\mu \| \gamma)$, and plugging Lemma 3 into (24) shows

$$
\begin{aligned}
\delta_{\text {Tal }}(\mu) & \geq \mathrm{D}(\mu \| \gamma) \int_{0}^{1}(2 t-1) \frac{\left(\mathrm{C}_{\mathrm{p}}(\mu)+1\right) t}{\left(\mathrm{C}_{\mathrm{p}}(\mu)-1\right) t+1} d t \\
& =\mathrm{D}(\mu \| \gamma) \frac{\left(\mathrm{C}_{\mathrm{p}}(\mu)+1\right)\left(2-2 \mathrm{C}_{\mathrm{p}}(\mu)+\left(\mathrm{C}_{\mathrm{p}}(\mu)+1\right) \ln \left(\mathrm{C}_{\mathrm{p}}(\mu)\right)\right)}{\left(\mathrm{C}_{\mathrm{p}}(\mu)-1\right)^{3}}
\end{aligned}
$$

where the equality relies on the fact

$$
\begin{aligned}
& \frac{d}{d t} \frac{\left(\mathrm{C}_{\mathrm{p}}(\mu)+1\right)\left(\left(\mathrm{C}_{\mathrm{p}}(\mu)-1\right) t\left(\mathrm{C}_{\mathrm{p}}(\mu)(t-1)-1-t\right)+\left(\mathrm{C}_{\mathrm{p}}(\mu)+1\right) \ln \left(\left(\mathrm{C}_{\mathrm{p}}(\mu)-1\right) t+1\right)\right)}{\left(\mathrm{C}_{\mathrm{p}}(\mu)-1\right)^{3}} \\
& \quad=(2 t-1) \frac{\left(\mathrm{C}_{\mathrm{p}}(\mu)+1\right) t}{\left(\mathrm{C}_{\mathrm{p}}(\mu)-1\right) t+1} .
\end{aligned}
$$

The proof is complete.

\subsection{Measures with small covariance}

Here we work under the assumption $\operatorname{Tr}(\operatorname{Cov}(\mu)) \leq d$ and prove Theorem 3 .

Proof of Theorem 3. Denote $c_{\mu}=\mathbb{E}\left[\left\|v_{1 / 2}\right\|_{2}^{2}\right]$. We begin by considering the case $c_{\mu} \leq \mathrm{D}(\mu \| \gamma)$. In this case, (23) shows

$$
\delta_{\text {Tal }}(\mu) \geq \frac{1}{4} \mathrm{D}(\mu \| \gamma)
$$


In the other case, $c_{\mu}>\mathrm{D}(\mu \| \gamma)$ and Lemma 2, along with (24), gives

$$
\begin{aligned}
\delta_{\text {Tal }}(\mu) & \geq 2 d \int_{0}^{1} \frac{c_{\mu}(2 t-1)}{c_{\mu}(1-2 t)+2 d} d t \\
& =\left.2 d\left(\frac{-d \ln \left(c_{\mu}+2 d-2 c_{\mu} t\right)-c_{\mu} t}{c_{\mu}}\right)\right|_{0} ^{1} \\
& =\frac{2 d\left(d \ln \left(2 d+c_{\mu}\right)-d \ln \left(2 d-c_{\mu}\right)-c_{\mu}\right)}{c_{\mu}} \\
& =2 d\left(\frac{2 d \operatorname{coth}^{-1}\left(\frac{2 d}{c_{\mu}}\right)}{c_{\mu}}-1\right) .
\end{aligned}
$$

Note that (20) implies $c_{\mu} \leq 2 d$, so the above is well defined. Also, for any $x \geq 1$, we have the inequality $\operatorname{coth}^{-1}(x) \cdot x-1 \geq \frac{1}{3 x^{2}}$, applying it to the previous bound then gives

$$
\delta_{\mathrm{Tal}}(\mu) \geq \frac{c_{\mu}^{2}}{6 d}>\frac{\mathrm{D}(\mu \| \gamma)^{2}}{6 d} .
$$

We can get a dimension free bound by considering directions $v \in \mathbb{R}^{d}$ in which $\operatorname{Cov}(\mu)$ is strictly smaller than the identity. For this we use Lemma 1 to establish:

$$
\frac{d}{d t} \mathbb{E}\left[\Gamma_{t}\right]=\frac{\mathbb{E}\left[\Gamma_{t}\right]-\mathbb{E}\left[\Gamma_{t}^{2}\right]}{1-t} \preceq \frac{\mathbb{E}\left[\Gamma_{t}\right]-\mathbb{E}\left[\Gamma_{t}\right]^{2}}{1-t} .
$$

Fix $v \in \mathbb{R}^{d}$, a unit vector, and define $f(t)=\left\langle v, \mathbb{E}\left[\Gamma_{t}\right] v\right\rangle$. As $\mathbb{E}\left[\Gamma_{t}\right]$ is symmetric, by CauchySchwartz

$$
\left\langle v, \mathbb{E}\left[\Gamma_{t}\right] v\right\rangle^{2} \leq\left\langle v, \mathbb{E}\left[\Gamma_{t}\right]^{2} v\right\rangle .
$$

This implies

$$
\frac{d}{d t} f(t) \leq \frac{f(t)(1-f(t))}{1-t}
$$

If $\left\langle v, \mathbb{E}\left[\Gamma_{0}\right] v\right\rangle=\lambda$, from Gronwall's inequality we get

$$
\left\langle v, \mathbb{E}\left[\Gamma_{t}\right] v\right\rangle \leq \frac{\lambda}{(\lambda-1) t+1}
$$

Using this, we prove Theorem 2.

Proof of Theorem 2. For $\lambda_{i}<1$, let $w_{i}$ be the unit eigenvector of $\operatorname{Cov}(\mu)$, corresponding to $\lambda_{i}$. From (25) we deduce, for every $t \in[0,1]$,

$$
0 \leq\left\langle w_{i}, \mathbb{E}\left[\Gamma_{t}\right] w_{i}\right\rangle \leq 1
$$

We now observe that as $v_{t}$ is a martingale, and since $\mu$ is centered, it must hold that $v_{0}=0$, almost surely. Combining this with (20) shows $\mathbb{E}\left[\Gamma_{0}\right]=\operatorname{Cov}(\mu)$ and in particular

$$
\left\langle w_{i}, \mathbb{E}\left[\Gamma_{0}\right] w_{i}\right\rangle=\lambda_{i}
$$


Using (25) and the fact that $\mathbb{E}\left[\Gamma_{t}\right]$ is symmetric, we obtain:

$$
t \frac{\left\langle w_{i}, \mathbb{E}\left[\left(\mathrm{I}_{d}-\Gamma_{t}\right)^{2}\right] w_{i}\right\rangle}{1-t} \geq t \frac{\left(\left\langle w_{i}, \mathbb{E}\left[\mathrm{I}_{d}-\Gamma_{t}\right] w_{i}\right\rangle\right)^{2}}{1-t} \geq \frac{t\left(1-\frac{\lambda_{i}}{\left(\lambda_{i}-1\right) t+1}\right)^{2}}{1-t}=t(1-t)\left(\frac{\lambda_{i}-1}{\left(\lambda_{i}-1\right) t+1}\right)^{2} .
$$

So, by (19),

$$
\begin{aligned}
\delta_{\text {Tal }}(\mu) & \geq \operatorname{Tr} \int_{0}^{1} t \cdot \frac{\mathbb{E}\left[\left(\mathrm{I}_{d}-\Gamma_{t}\right)^{2}\right]}{1-t} d t \geq \sum_{i=1}^{d} \mathbb{1}_{\left\{\lambda_{i}<1\right\}} \int_{0}^{1} t \cdot \frac{\left\langle v_{i}, \mathbb{E}\left[\left(\mathrm{I}_{d}-\Gamma_{t}\right)^{2}\right] v_{i}\right\rangle}{1-t} d t \\
& \geq \sum_{i=1}^{d} \mathbb{1}_{\left\{\lambda_{i}<1\right\}} \int_{0}^{1} t(1-t)\left(\frac{\lambda_{i}-1}{\left(\lambda_{i}-1\right) t+1}\right)^{2} d t \\
& =\sum_{i=1}^{d} \frac{2\left(1-\lambda_{i}\right)+\left(\lambda_{i}+1\right) \log \left(\lambda_{i}\right)}{\lambda_{i}-1} \mathbb{1}_{\left\{\lambda_{i}<1\right\}} .
\end{aligned}
$$

\subsection{Stability with respect to Gaussian mixtures}

In this section we prove Theorem 5. Our proof is based on [12], but we use our framework to give an improved analysis. To control the relative entropy we will use a specialized case of the bound given in [14]. We supply here a sketch of the proof for completeness.

Lemma 4. Let $H_{t}$ be an $\mathcal{F}_{t}$-adapted matrix-valued processes and let $N_{t}$ be defined by

$$
N_{t}=\int_{0}^{t} H_{s} d B_{s}
$$

Suppose that $\tilde{H}_{t}$ is such that for some $t_{0} \in[0,1]$ :

1. $\tilde{H}_{t}=H_{t}$ almost surely, for $t<t_{0}$.

2. For $t \geq t_{0}, \tilde{H}_{t}$ is deterministic and $\tilde{H}_{t} \succ \mathrm{I}_{d}$.

Then, if $M_{t}$ is defined by

$$
M_{t}=\int_{0}^{t} \tilde{H}_{s} d B_{s}
$$

we have

$$
\operatorname{Ent}\left(N_{1} \| M_{1}\right) \leq \operatorname{Tr} \int_{t_{0}}^{1} \frac{\mathbb{E}\left[\left(H_{t}-\tilde{H}_{t}\right)^{2}\right]}{1-t} d t .
$$

Proof. Define the process

$$
Y_{t}=\int_{0}^{t} \tilde{H}_{s} d B_{s}+\int_{0}^{t} \int_{0}^{s} \frac{H_{r}-\tilde{H}_{r}}{1-r} d B_{r} d s
$$


Denote $u_{t}=\int_{0}^{t} \frac{H_{s}-\tilde{H}_{s}}{1-s} d B_{s}$, so that, $d Y_{t}=\tilde{H}_{t} d B_{t}+u_{t} d t$, and, by assumption $u_{t}=0$, whenever $t<t_{0}$. It follows that $Y_{t}=M_{t}$ for $t<t_{0}$ and that, using Fubini's theorem, $Y_{1}=N_{1}$. Indeed,

$$
Y_{1}=\int_{0}^{1} \tilde{H}_{t} d B_{t}+\int_{0}^{1} \int_{0}^{t} \frac{H_{s}-\tilde{H}_{s}}{1-s} d B_{s} d t=\int_{0}^{1} \tilde{H}_{t} d B_{t}+\int_{0}^{1}\left(H_{t}-\tilde{H}_{t}\right) d B_{t}=N_{1} .
$$

We denote now by $P$, the measure under which $B$ is a Brownian motion. If

$$
\mathcal{E}:=\exp \left(-\int_{0}^{1} \tilde{H}_{t}^{-1} u_{t} d B_{t}-\frac{1}{2} \int_{0}^{1}\left\|\tilde{H}_{t}^{-1} u_{t}\right\|^{2} d t\right)
$$

and we define the tilted measure $Q=\mathcal{E} P$, then by Girsanov's theorem, $\tilde{B}_{t}=B_{t}+\int_{0}^{t} \tilde{H}_{s}^{-1} u_{s} d s$ is a Brownian motion under $Q$ and we have the representation

$$
Y_{t}=\int_{0}^{t} \tilde{H}_{s} d \tilde{B}_{s}
$$

If $t<t_{0}$, then as $u_{t}=0$, we have $\tilde{B}_{t}=B_{t}$ and $Y_{t_{0}}$ has the same law under $Q$ and under $P$, which is the law of $M_{t_{0}}$. Moreover, for $t \geq t_{0}, \tilde{H}_{t}$ is deterministic. Therefore, it is also true that the law of

$$
Y_{t_{0}}+\int_{t_{0}}^{1} \tilde{H}_{t} d \tilde{B}_{t}
$$

under $Q$ and the law of

$$
Y_{t_{0}}+\int_{t_{0}}^{1} \tilde{H}_{t} d B_{t}
$$

under $P$ coincide. We thus conclude that, under $Q, Y_{1}$ has the same law as $M_{1}$ under $P$. In particular, if $\rho$ is the density of $Y_{1}$ with respect to $M_{1}$, this implies

$$
1=\mathbb{E}_{P}\left[\rho\left(M_{1}\right)\right]=\mathbb{E}_{Q}\left[\rho\left(Y_{1}\right)\right]=\mathbb{E}_{P}\left[\rho\left(Y_{1}\right) \mathcal{E}\right] .
$$

By Jensen's inequality, under $P$,

$$
0=\ln \left(\mathbb{E}\left[\rho\left(Y_{1}\right) \mathcal{E}\right]\right) \geq \mathbb{E}\left[\ln \left(\rho\left(Y_{1}\right) \mathcal{E}\right)\right]=\mathbb{E}\left[\ln \left(\rho\left(Y_{1}\right)\right)\right]+\mathbb{E}[\ln (\mathcal{E})]
$$

But,

$$
\begin{aligned}
-\mathbb{E}[\ln (\mathcal{E})] & =\frac{1}{2} \int_{0}^{1} \mathbb{E}\left[\left\|\tilde{H}_{t}^{-1} u_{t}\right\|^{2}\right] d t \leq \int_{t_{0}}^{1} \mathbb{E}\left[\left\|u_{t}\right\|^{2}\right] d t \\
& =\operatorname{Tr} \int_{t_{0}}^{1} \int_{t_{0}}^{s} \frac{\mathbb{E}\left[\left(H_{s}-\tilde{H}_{s}\right)^{2}\right]}{(1-s)^{2}} d s d t=\operatorname{Tr} \int_{t_{0}}^{1} \int_{s}^{1} \frac{\mathbb{E}\left[\left(H_{s}-\tilde{H}_{s}\right)^{2}\right]}{(1-s)^{2}} d t d s \\
& =\operatorname{Tr} \int_{t_{0}}^{1} \frac{\mathbb{E}\left[\left(H_{s}-\tilde{H}_{s}\right)^{2}\right]}{1-s} d s
\end{aligned}
$$


and, from (26)

$$
\mathbb{E}_{P}\left[\ln \left(\rho\left(Y_{1}\right)\right)\right]=\operatorname{Ent}\left(N_{1} \| M_{1}\right),
$$

which concludes the proof.

Remark 7. In order to apply Girsanov's theorem in the proof above, one must also require some integrability condition from the drift $u_{t}$. It will suffice to assume

$$
\int_{0}^{1} \mathbb{E}\left[\left\|\tilde{H}_{t}^{-1} u_{t}\right\|^{2}\right] d t<\infty
$$

Indeed, if $\int_{0}^{1}\left\|\tilde{H}_{t}^{-1} u_{t}\right\|^{2} d t$ is uniformly bounded, then Novikov's criterion applies. The general case may then be obtained by an approximation argument (see [25, Proposition 1] for more details). In our application below this condition will be satisfied.

We are now in a position to prove that stability with respect to Gaussian mixtures holds in relative entropy.

Proof of Theorem 5. Fix $t_{0} \in[0,1]$, by (19) we get

$$
\delta_{\text {Tal }}(\mu) \geq t_{0} \operatorname{Tr} \int_{t_{0}}^{1} \frac{\mathbb{E}\left[\left(\mathrm{I}_{d}-\Gamma_{t}\right)^{2}\right]}{1-t} d t .
$$

Define the matrix-valued process

$$
\tilde{\Gamma}_{t}=\left\{\begin{array}{ll}
\Gamma_{t} & 0 \leq t<t_{0} \\
\frac{1-t_{0}}{t_{0}(t-2)+1} I_{d} & t_{0} \leq t \leq 1
\end{array},\right.
$$

and the martingale

$$
M_{t}=\int_{0}^{t} \tilde{\Gamma}_{s} d B_{s}
$$

One may verify that

$$
\int_{t_{0}}^{1}\left(\frac{1-t_{0}}{t_{0}(t-2)+1}\right)^{2} d t=1
$$

which implies, $M_{1}-M_{t_{0}}=\int_{t_{0}}^{1} \tilde{\Gamma}_{t}\left(M_{t}\right) d B_{t} \sim \gamma$. Also, from (13),

$$
M_{t}=\int_{0}^{t_{0}} \tilde{\Gamma}_{t} d B_{t}=\int_{0}^{t_{0}} \Gamma_{t} d B_{t}=\mathbb{E}\left[X_{1} \mid \mathcal{F}_{t_{0}}\right] .
$$

If $\nu_{t_{0}}$ is the law of $\mathbb{E}\left[X_{1} \mid \mathcal{F}_{t_{0}}\right]$, then since $\left\{B_{s}\right\}_{s>t_{0}}$ is independent from $\mathbb{E}\left[X_{1} \mid \mathcal{F}_{t_{0}}\right]$, we have that $\nu_{t_{0}} * \gamma$ is the law of $M_{1}$. 
We now invoke Lemma 4 with the process $\mathbb{E}\left[X_{1} \mid \mathcal{F}_{t}\right]$ as $N_{t}$. Since $\tilde{\Gamma}_{t}$ meets the conditions of the lemma, we get

$$
\begin{aligned}
\mathrm{D}\left(X_{1} \| M_{1}\right)=\mathrm{D}\left(\mu \| \nu_{t_{0}} * \gamma\right) & \leq \operatorname{Tr} \int_{t_{0}}^{1} \frac{\mathbb{E}\left[\left(\Gamma_{t}-\tilde{\Gamma}_{t}\right)^{2}\right]}{1-t} d t \\
& \leq 2 \operatorname{Tr} \int_{t_{0}}^{1} \frac{\mathbb{E}\left[\left(\Gamma_{t}-\mathrm{I}_{d}\right)^{2}\right]}{1-t} d t+2 \operatorname{Tr} \int_{t_{0}}^{1} \frac{\mathbb{E}\left[\left(\tilde{\Gamma}_{t}-\mathrm{I}_{d}\right)^{2}\right]}{1-t} d t .
\end{aligned}
$$

Observe that by showing that the above integrals are finite we will also verify the integrability condition from Remark 7. Applying (27),

$$
2 \operatorname{Tr} \int_{t_{0}}^{1} \frac{\mathbb{E}\left[\left(\Gamma_{t}-\mathrm{I}_{d}\right)^{2}\right]}{1-t} d t \leq 2 \frac{\delta_{\text {Tal }}(\mu)}{t_{0}} .
$$

To bound the second term we calculate

$$
\begin{aligned}
2 \operatorname{Tr} \int_{t_{0}}^{1} \frac{\mathbb{E}\left[\left(\tilde{\Gamma}_{t}-\mathrm{I}_{d}\right)^{2}\right]}{1-t} d t & =2 d \int_{t_{0}}^{1} \frac{\left(\frac{1-t_{0}}{t_{0}(t-2)+1}-1\right)^{2}}{1-t} d t \\
& =\left.2 d\left(-\ln \left(1+t_{0}(t-2)\right)-\frac{1-t_{0}}{2\left(t-t_{0}\right)+1}\right)\right|_{t_{0}} ^{1} \\
& =2 d\left(\ln \left(1-t_{0}\right)+\frac{t_{0}}{1-t_{0}}\right) .
\end{aligned}
$$

Combining the last displays, we get

$$
\mathrm{D}\left(\mu \| \nu_{t_{0}} * \gamma\right) \leq 2\left(\frac{\delta_{\mathrm{Tal}}(\mu)}{t_{0}}+d\left(\ln \left(1-t_{0}\right)+\frac{t_{0}}{1-t_{0}}\right)\right) .
$$

Suppose that $\delta_{\mathrm{Tal}}(\mu) \geq d$, then choosing $t_{0}=\frac{1}{2}$ gives

$$
\frac{\mathrm{D}\left(\mu|| \nu_{t_{0}} * \gamma\right)}{6} \leq \delta_{\mathrm{Tal}}(\mu)
$$

Otherwise, $\delta_{\mathrm{Tal}}(\mu)<d$ and we choose $t_{0}=\left(\frac{\delta_{\mathrm{Tal}}(\mu)}{d}\right)^{\frac{1}{3}} \leq \frac{1}{2}$. A second order approximation, shows that for $s \in\left[0, \frac{1}{2}\right]$,

$$
\ln (1-s)+\frac{s}{1-s} \leq 2 s^{2} .
$$

Hence, for the above choice of $t_{0}$,

$$
\mathrm{D}\left(\mu \| \nu_{t_{0}} * \gamma\right) \leq 2 \frac{\delta_{\text {Tal }}(\mu)}{t_{0}}+4 d t_{0}^{2}=3 \delta_{\text {Tal }}(\mu)^{\frac{2}{3}} d^{\frac{1}{3}} .
$$

This implies

$$
\frac{1}{3 \sqrt{3}} \frac{\mathrm{D}\left(\mu \| \nu_{t_{0}} * \gamma\right)^{\frac{3}{2}}}{\sqrt{d}} \leq \delta_{\mathrm{Tal}}(\mu)
$$


which is the desired claim. Finally, by the law of total variance, it is immediate that

$$
\operatorname{Cov}\left(\nu_{t_{0}}\right) \preceq \operatorname{Cov}(\mu) .
$$

\section{An application to Gaussian concentration}

We now show that our stability bounds imply an improved Gaussian concentration inequality for concave functions.

Corollary 8. Let $f$ be a concave function and $G \sim \gamma$ in $\mathbb{R}^{d}$. Suppose that $f$ is even, then for any $t \geq 0$,

$$
\mathbb{P}(f(G) \geq t) \leq e^{-\frac{4 t^{2}}{7 \mathbb{E}\left[\|\nabla f(G)\|_{2}^{2}\right]}} .
$$

Before proving the result we mention that our proof follows the one presented in [29]. We use Theorem 1 to improve the constant obtained there. One should also compare the corollary to the main result of [28] which used Ehrhard's inequality in order to show that $\mathbb{E}\left[\|\nabla f(G)\|_{2}^{2}\right]$ may be replaced by the smaller quantity $\operatorname{Var}(f(G))$, at the cost of a worse constant in the exponent.

The assumption that $f$ is even is used here for simplicity and could be relaxed.

Proof of Corollary 8. For $\lambda>0$, denote the measure $\nu_{\lambda}=\frac{e^{\lambda f}}{\mathbb{E}_{\gamma}\left[e^{\lambda f}\right]} d \gamma$ and let $(X, Y)$ be a random vector in $\mathbb{R}^{2 d}$ which is a realization of the optimal coupling between $\nu_{\lambda}$ and $\gamma$. That is, $X \sim \nu_{\lambda}, Y \sim \gamma$ and

$$
\mathcal{W}_{2}\left(\nu_{\lambda}, \gamma\right)=\sqrt{\mathbb{E}\left[\|X-Y\|_{2}^{2}\right]}
$$

As $f$ is concave, we have by using Cauchy-Schwartz:

$$
\begin{aligned}
\mathbb{E}_{\nu_{\lambda}}[\lambda f]-\mathbb{E}_{\gamma}[\lambda f] & \leq \mathbb{E}[\langle\nabla \lambda f(Y), X-Y\rangle] \leq \sqrt{\lambda^{2} \mathbb{E}\left[\|\nabla f(Y)\|_{2}^{2}\right]} \sqrt{\mathbb{E}\left[\|X-Y\|_{2}^{2}\right]} \\
& =\sqrt{\lambda^{2} \mathbb{E}_{\gamma}\left[\|\nabla f\|_{2}^{2}\right]} \mathcal{W}_{2}\left(\nu_{\lambda}, \gamma\right)
\end{aligned}
$$

Since $f$ is concave, $\nu_{\lambda}$ has a log-concave density with respect to the standard Gaussian. For such measures, Brascamp-Lieb's inequality ( [5]) dictates that $\mathrm{C}_{\mathrm{p}}\left(\nu_{\lambda}\right) \leq 1$. Note that

$$
\frac{(x+1)(2-2 x+(x+1) \ln (x)}{(x-1)^{3}} \geq \frac{1}{3}, \text { whenever } x \in[0,1] .
$$

In this case, since $f$ is even and $\nu_{\lambda}$ is centered, Theorem 1 gives us,

$$
\delta_{\text {Tal }}\left(\nu_{\lambda}\right) \geq \frac{1}{4} \mathrm{D}\left(\nu_{\lambda} \| \gamma\right)
$$

which is equivalent to

$$
\mathcal{W}_{2}^{2}\left(\nu_{\lambda}, \gamma\right) \leq \frac{7}{4} \mathrm{D}\left(\nu_{\lambda} \| \gamma\right)
$$

Combining this with (28) and the assumption, $\mathbb{E}_{\gamma}[\lambda f]=0$, yields

$$
\mathbb{E}_{\nu_{\lambda}}[\lambda f] \leq \sqrt{\lambda^{2} \frac{7}{4} \mathbb{E}_{\gamma}\left[\|\nabla f\|_{2}^{2}\right] \mathrm{D}\left(\nu_{\lambda} \| \gamma\right)}
$$


For any $x, y \geq 0$ we have the inequality,

$$
\sqrt{x y} \leq \frac{x}{4}+y
$$

Observe as well that

$$
\mathrm{D}\left(\nu_{\lambda} \| \gamma\right)=\mathbb{E}_{\nu_{\lambda}}[\lambda f]-\ln \left(\mathbb{E}_{\gamma}\left[e^{\lambda f}\right]\right)
$$

Thus,

$$
\ln \left(\mathbb{E}_{\gamma}\left[e^{\lambda f}\right]\right) \leq \lambda^{2} \frac{7}{16} \mathbb{E}_{\gamma}\left[\|\nabla f\|_{2}^{2}\right] .
$$

By Markov's inequality, for any $\lambda, t>0$

$$
\mathbb{P}(f(G) \geq t)=\mathbb{P}\left(e^{\lambda f(G)} \geq e^{\lambda t}\right) \leq \mathbb{E}_{\gamma}\left[e^{\lambda f}\right] e^{-\lambda t} \leq \exp \left(\lambda^{2} \frac{7}{16} \mathbb{E}_{\gamma}\left[\|\nabla f\|_{2}^{2}\right]-\lambda t\right) .
$$

We now optimize over $\lambda$ to obtain,

$$
\mathbb{P}(f(G) \geq t) \leq e^{\left.-\frac{4 t^{2}}{7 \mathbb{E}_{\gamma}\left[\|\nabla f\|_{2}^{2}\right.}\right]}
$$

\section{References}

[1] Marco Barchiesi, Alessio Brancolini, and Vesa Julin. Sharp dimension free quantitative estimates for the Gaussian isoperimetric inequality. Ann. Probab., 45(2):668-697, 2017.

[2] S. G. Bobkov, N. Gozlan, C. Roberto, and P.-M. Samson. Bounds on the deficit in the logarithmic Sobolev inequality. J. Funct. Anal., 267(11):4110-4138, 2014.

[3] Christer Borell. The Ehrhard inequality. C. R. Math. Acad. Sci. Paris, 337(10):663-666, 2003.

[4] A. A. Borovkov and S. A. Utev. An inequality and a characterization of the normal distribution connected with it. Teor. Veroyatnost. i Primenen., 28(2):209-218, 1983.

[5] Herm Jan Brascamp and Elliott H. Lieb. On extensions of the Brunn-Minkowski and Prékopa-Leindler theorems, including inequalities for log concave functions, and with an application to the diffusion equation. J. Functional Analysis, 22(4):366-389, 1976.

[6] Eric A. Carlen, Maria C. Carvalho, Jonathan Le Roux, Michael Loss, and Cédric Villani. Entropy and chaos in the Kac model. Kinet. Relat. Models, 3(1):85-122, 2010.

[7] A. Cianchi, N. Fusco, F. Maggi, and A. Pratelli. On the isoperimetric deficit in Gauss space. Amer. J. Math., 133(1):131-186, 2011.

[8] Dario Cordero-Erausquin. Transport inequalities for log-concave measures, quantitative forms, and applications. Canad. J. Math., 69(3):481-501, 2017.

[9] Thomas A. Courtade, Max Fathi, and Ashwin Pananjady. Quantitative stability of the entropy power inequality. IEEE Trans. Inform. Theory, 64(8):5691-5703, 2018. 
[10] Thomas M. Cover and Joy A. Thomas. Elements of information theory. Wiley-Interscience [John Wiley \& Sons], Hoboken, NJ, second edition, 2006.

[11] Ronen Eldan and James R. Lee. Regularization under diffusion and anticoncentration of the information content. Duke Math. J., 167(5):969-993, 2018.

[12] Ronen Eldan, Joseph Lehec, and Yair Shenfeld. Stability of the logarithmic Sobolev inequality via the Föllmer process. arXiv preprint arXiv:1903.04522, 2019.

[13] Ronen Eldan and Dan Mikulincer. Stability of the Shannon-Stam inequality via the Föllmer process. arXiv preprint arXiv:1903.07140, 2019.

[14] Ronen Eldan, Dan Mikulincer, and Alex Zhai. The CLT in high dimensions: quantitative bounds via martingale embedding. arXiv preprint arXiv:1806.09087, 2018.

[15] Max Fathi, Emanuel Indrei, and Michel Ledoux. Quantitative logarithmic Sobolev inequalities and stability estimates. Discrete Contin. Dyn. Syst., 36(12):6835-6853, 2016.

[16] F. Feo, E. Indrei, M. R. Posteraro, and C. Roberto. Some remarks on the stability of the log-Sobolev inequality for the Gaussian measure. Potential Anal., 47(1):37-52, 2017.

[17] H. Föllmer. An entropy approach to the time reversal of diffusion processes. In Stochastic differential systems (Marseille-Luminy, 1984), volume 69 of Lect. Notes Control Inf. Sci., pages 156-163. Springer, Berlin, 1985.

[18] H. Föllmer. Time reversal on Wiener space. In Stochastic processes-mathematics and physics (Bielefeld, 1984), volume 1158 of Lecture Notes in Math., pages 119-129. Springer, Berlin, 1986.

[19] Ivan Gentil, Christian Léonard, and Luigia Ripani. About the analogy between optimal transport and minimal entropy. Ann. Fac. Sci. Toulouse Math. (6), 26(3):569-601, 2017.

[20] N. Gozlan and C. Léonard. Transport inequalities. A survey. Markov Process. Related Fields, 16(4):635-736, 2010.

[21] Leonard Gross. Logarithmic Sobolev inequalities. Amer. J. Math., 97(4):1061-1083, 1975.

[22] Alexander V. Kolesnikov and Egor D. Kosov. Moment measures and stability for Gaussian inequalities. Theory Stoch. Process., 22(2):47-61, 2017.

[23] Michel Ledoux. The concentration of measure phenomenon, volume 89 of Mathematical Surveys and Monographs. American Mathematical Society, Providence, RI, 2001.

[24] Michel Ledoux, Ivan Nourdin, and Giovanni Peccati. A Stein deficit for the logarithmic Sobolev inequality. Sci. China Math., 60(7):1163-1180, 2017.

[25] Joseph Lehec. Representation formula for the entropy and functional inequalities. Ann. Inst. Henri Poincaré Probab. Stat., 49(3):885-899, 2013.

[26] Elchanan Mossel and Joe Neeman. Robust dimension free isoperimetry in Gaussian space. Ann. Probab., 43(3):971-991, 2015. 
[27] Bernt $\varnothing$ ksendal. Stochastic differential equations. Universitext. Springer-Verlag, Berlin, sixth edition, 2003. An introduction with applications.

[28] Grigoris Paouris and Petros Valettas. A Gaussian small deviation inequality for convex functions. Ann. Probab., 46(3):1441-1454, 2018.

[29] Paul-Marie Samson. Concentration of measure inequalities for Markov chains and $\Phi$ mixing processes. Ann. Probab., 28(1):416-461, 2000.

[30] M. Talagrand. Transportation cost for Gaussian and other product measures. Geom. Funct. Anal., 6(3):587-600, 1996.

[31] Cédric Villani. Optimal transport: old and new, volume 338. Springer Science \& Business Media, 2008. 